\title{
Computational Prediction of Primary Breakup in Fuel Spray Nozzles for Aero-Engine Combustors
}

\author{
Thilo F. Dauch*, Samuel Braun ${ }^{2}$, Lars Wieth ${ }^{3}$, Geoffroy Chaussonnet ${ }^{4}$, Marc C. Keller ${ }^{5}$, \\ Rainer Koch ${ }^{6}$, Hans-Jörg Bauer ${ }^{7}$ \\ Institut für Thermische Strömungsmaschinen (ITS), \\ Karlsruhe Institute of Technology (KIT), Germany \\ *Corresponding author: thilo.dauch@kit.edu
}

\begin{abstract}
Primary breakup of liquid fuel in the vicinity of fuel spray nozzles as utilized in aero-engine combustors is numerically investigated. As grid based methods exhibit a variety of disadvantages when it comes to the prediction of multiphase flows, the "Smoothed Particle Hydrodynamics" (SPH)-method is employed. The eligibility of the method to analyze breakup of fuel has been demonstrated in recent publications by Braun et al, Dauch et al and Koch et al $[1,2,3,4]$. In the current paper a methodology for the investigation of the two-phase flow in the vicinity of fuel spray nozzles at typical operating conditions is proposed. Due to lower costs in terms of computing time, 2D predictions are desired. However, atomization of fluids is inherently three dimensional. Hence, differences between 2D and 3D predictions are to be expected. In course of this study, predictions in 2D and based on a 3D sector are presented. Differences in terms of gaseous flow, ligament shape and mixing are assessed.
\end{abstract}

\section{Keywords}

Multiphase Flows - Fuel Atomization - Smoothed Particle Hydrodynamics - Aero-Engine - Combustor

\section{Introduction}

Aiming at a reduction of pollutant emissions of air-traffic, academia and industry both invest in research to investigate processes causing the formation of pollutants of aero-engine combustors. One aspect influencing the formation of pollutants is the quality of the injected fuel spray and its placement within the gaseous flow field of the combustion chamber.

Because of limited optical access and challenging thermodynamic conditions, experimental studies are costly and cannot provide detailed information about breakup of the fuel in the close vicinity of fuel injectors. Hence, numerical investigations analyzing the local two-phase flow are desired.

At the "Institut für Thermische Strömungsmaschinen" (ITS) a numerical code based on the Lagrangian "Smoothed Particle Hydrodynamics" (SPH)-method has been developed by Koch, Hoefler and Braun [5, 4]. The objective is to predict the liquid fuel breakup in the close vicinity of the fuel spray nozzle. Conventional grid based methods exhibit a variety of inherent shortcomings, which can be overcome by a fully Lagrangian approach. In recent publications the potential of the code in terms of two-phase flow predictions has been demonstrated successfully by Braun et al, Dauch et al and Keller et al [1, 3, 6].

Current state-of-the art methods for combustor design do not take into account the details of primary breakup. Correlations based on empirical studies are employed to impose droplet initial conditions for subsequent EulerLagrangian CFD predictions. These correlations must be tuned to each individual setup and need to be calibrated. Most of the correlations are valid only for low pressures and temperatures. A detailed simulation of primary breakup can overcome these shortcomings. It might provide transient droplet initial conditions, which can be used as input for subsequent Euler-Lagrangian predictions. Even if SPH computations are too costly for every day design studies, they can help deriving simplified primary breakup models such as presented by Chausonnet et al [7], that are efficient and accurate enough to be integrated in conventional CFD-codes.

The current study demonstrates, how SPH predictions can be used to investigate the details of primary breakup at fuel spray nozzles typically utilized in aero-engines at realistic operating conditions. As fuel spray atomization is inherently three dimensional, predictions in 3D are desired. Because of the lower costs for 2D predictions, design studies in 2D are preferred if possible. However, deviations between 2D and 3D predictions are to be expected. To identify the deviations this study will address the comparison between $2 \mathrm{D}$ and $3 \mathrm{D}$ results.

\section{Numerical Method}

The original purpose of the "Smoothed Particle Hydrodynamics"(SPH)-method is the analysis of phenomena occurring in astrophysics as presented by Gingold et al and Lucy [8, 9]. Nowadays, SPH is employed for the analysis of different problems in science and engineering as demonstrated by Chaussonnet et al, Dauch et al and Keller et al $[10,3,6]$. In contrast to conventional grid based CFD methods, SPH is a fully Lagrangian method and does not require a computational mesh. The flow domain is discretized by means of moving discretization points, referred to as "particles".

In grid based methods the location of the phase interface depends on the definition of the limited volume fraction resulting in reduced accuracy. The phase interface needs to be reconstructed, which is computationally expensive and prone to interface diffusion. 
Due to the Lagrangian character of the SPH method, phase interfaces of multi-phase flows are inherently advected and do not have to be reconstructed. Once particles are initialized as liquid, they remain liquid. Thus, the contour of the ligaments is uniquely defined by the location of the liquid particles. Hence, interface reconstruction is avoided. As primary breakup is entirely resolved, no breakup criterion is required. Additionally, particle methods can be parallelized more efficiently and therefore are less computationally expensive.

One drawback inherent to the method is its instability referred to as "Tensile Instability" as presented by Swegle et al [11]. Even though a variety of mitigations has been developed, which are summarized by Liu et al [12], the problem is still not solved in general. Another problem of SPH is the consistency issue, as described by Liu et al and Quinlan et al $[12,13]$. Both aspects are subject of ongoing research as described by Chaussonnet et al and Litvinov et al $[10,14]$. However, despite these known issues, SPH has been demonstrated to capture primary atomization with high accuracy as presented by Koch et al [4].

The fluid flow is modeled by means of the Navier-Stokes equations and corresponding boundary conditions. In the present work the flow is considered to be isothermal. Hence, the energy equation is not considered here. The substantial derivatives of density and velocity are defined by continuity and momentum equation:

$$
\begin{aligned}
\frac{D \rho}{D t} & =-\rho(\vec{\nabla} \cdot \vec{v}), \\
\rho \frac{D \vec{v}}{D t} & =-\vec{\nabla} p+\vec{\nabla} \cdot \tau+\rho \vec{f} .
\end{aligned}
$$

In order to illustrate the formalism of the SPH-method, the formulation of any function $f(\vec{r})$ in terms of the convolution integral is utilized:

$$
f(\vec{r})=\int f\left(\overrightarrow{r^{\prime}}\right) \delta\left(\vec{r}-\overrightarrow{r^{\prime}}\right) d \overrightarrow{r^{\prime}}
$$

Replacing the Dirac function by an approximation called kernel function $W\left(\vec{r}_{a}-\vec{r}_{b}, h\right)$ yields an approximated convolution integral. A variety of kernel functions exists as pointed out and discussed by Dehnen in [15]. In the present study a quintic kernel function is used. The typical shape of a kernel function is depicted in Fig. 1a. The magnitude $h$ is called the smoothing length. Depending on $h$, the so called radius of influence, exemplified in Fig. $1 \mathrm{~b}$, is varied.

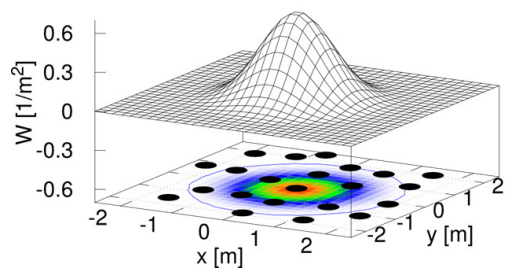

(a) Kernel Function [16]

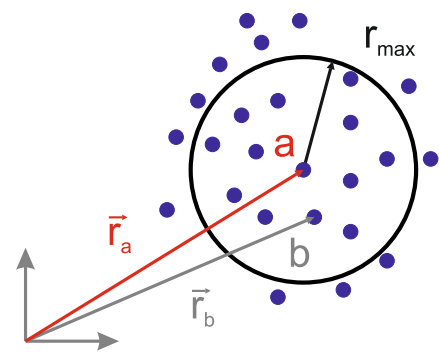

(b) Radius of Influence

Figure 1. SPH Methodology

Approximating the integral by a finite sum leads to a quadrature. Consequently, a scheme for the computation of derivatives can be determined as presented by Liu et al [12]:

$$
\begin{aligned}
\langle f\rangle_{a} & =\sum_{b} f\left(\vec{r}_{b}\right) W\left(\vec{r}_{a}-\vec{r}_{b}, h\right) V_{b}, \\
\langle\nabla \cdot f\rangle_{a} & =-\sum_{b} f\left(\vec{r}_{b}\right) \nabla W\left(\vec{r}_{a}-\vec{r}_{b}, h\right) V_{b} .
\end{aligned}
$$

The brackets \langle\rangle represent the SPH approximation. The index $a$ denominates the central particle and index $b$ any particle inside the radius of influence. The variable $V_{b}$ indicates the volume of each individual particle $b$. The resulting quadratures are the basic SPH schemes. Equation 4 demonstrates that any magnitude $\langle f\rangle_{a}$ at the position of particle $a$ can be determined in terms of a weighted sum $f$ over the function $f\left(r_{b}\right)$ of all other particles $b$ located inside the radius of influence.

By means of the quadratures, the terms on the right hand side of the Navier-Stokes equations as presented in Eq. 1 and 2 can be approximated. The substantial derivatives are computed and integrated in time by means of a predictor-corrector time integration scheme. In the present study, the density is not computed by means of the discretized continuity equation, but by the direct SPH scheme according to Colagrossi et al [17]:

$$
\langle\rho\rangle_{a}=m_{a} \sum_{b} W\left(\vec{r}_{a}-\vec{r}_{b}, h\right)
$$


The pressure gradient is computed as proposed by Monaghan in [18]:

$$
\left\langle-\frac{\vec{\nabla} p}{\rho}\right\rangle_{a}=-\frac{1}{\rho_{a}} \sum_{b} \frac{m_{b}}{\rho_{b}}\left(p_{a}+p_{b}\right) \vec{\nabla} W\left(\vec{r}_{a}-\vec{r}_{b}, h\right) .
$$

Viscosity is modeled by means of a viscosity model presented by Szewc [19]. These approximations conserve momentum and ensure stability in case of large density ratios. As equation of state the Tait-Volume-Equation according to Batchelor [20] is used:

$$
p=\frac{\rho_{n o m} c^{2}}{\kappa} \cdot\left[\left(\frac{V_{n o m}}{V}\right)^{\kappa}-1\right]+p_{\text {back }} .
$$

where $V$ is the volume and $p$ the pressure of each particle. The quantitiy $c$ is the speed of sound and $\kappa$ the polytropic exponent. Index nom designates the reference value of the corresponding quantity. The background pressure $p_{b a c k}$ is used in weakly compressible SPH to mitigate the inherent tensile instability of the SPH method. As to the assumptions of the weakly compressible approach, pressure depends only on density or volume, respectively. Hence, the equation of state is applied after the volume of each particle has been computed by means of Eq. 6 . Following Wieth et al [21], a modified version of the surface tension model by Adami et al [22] is employed. Static and dynamic contact angles are accurately predicted based on the prescribed surface tension coefficients. According to Young's equation, static contact angles of $60^{\circ}$ are recovered for the present case. In dynamic flows contact angles deviate from the static values. The current implementation takes this effect into account as a result of the balance of forces. The prediction of static and dynamic contact angles has been demonstrated and validated by Wieth et al [21].

As pointed out by Violeau and Rogers [23] three different types of wall treatment exist: Fictitious particles, repulsive functions and boundary integrals. The objective of all wall boundary treatments is to provide kernel support within the radius of influence of all fluid particles across the solid wall boundary. In the current implementation, prescribed dummy particles of at least three layers are used as solid wall boundary representation. Additionally, a repulsive function resembling the Lennard-Jones potential is employed as presented by Wieth et al [21] to avoid particle intrusion. By means of these wall treatments the no-slip condition at the wall is imposed.

In 3D simulations, the circumferential velocity component can easily be imposed the inlet. Centrifugal forces are inherently imposed by the swirling flow. In order to mimic the centrifugal forces in the 2D setup, a model aiming at a representation of centrifugal forces is incorporated. For each SPH particle entering the domain, the radius and the circumferential velocity is stored. In other words, the angular momentum of the particle entering the domain is recovered. When assuming the conservation of the angular momentum, radial equilibrium as well as incompressible fluids, the variation of the circumferential velocity of a particle can be determined as function of its actual radius. Then the corresponding centrifugal forces can be assigned. The model is presented previously by the author in [3].

\section{Fuel Spray Nozzle}

The fuel spray nozzle investigated in the present study consists of three annular, coaxial air ducts referred to as inner air, outer air and dome air according to the setup presented by Mansour et al [24]. All air flows exhibit a swirl component because of the arrangement of swirl generators in each duct as depicted in Fig. 2a. Because of the high angular momentum of all air flows, high centrifugal forces occur forcing the flow in radial direction. As the ratio of angular and axial momentum is large, it comes to vortex breakdown. A stagnation point (SP) is formed, in the vicinity of the middle axis as indicated in Fig. $2 \mathrm{~b}$.

The main objective of the dome air flow is to shape the recirculation zone inside the combustor. In between the inner and the outer air duct, fuel is supplied through an annular, coaxial slot across the whole perimeter. The fuel is released into the shear zone between inner and outer air. The shear stress imposed by the air will act against the surface tension and finally the fuel will be disintegrated.

Local recirculation zones are to be expected downstream the bluff edges of each shroud as indicated in Fig. $2 b$. The flows of inner and outer air form a stream tube. Per definition, no mass transfer takes place across the boundaries of the tubes. These boundaries are indicated as dashed lines in Fig. 2b.

\section{Computational Setup}

Main objective of this study is the analysis of the multi-phase flow in the vicinity of the prefilming edge in 2D and 3D. The focus is on the interaction of the inner air flow with the fuel in the fuel supply duct, the formation of ligaments and their disintegration. Dispersion of the fuel droplets, their placement inside the combustor and secondary breakup are not the objective of the present study.

The numerical domain is a three dimensional sector based on the axisymmetric cross-section depicted in Fig. 3a. There are two inlet boundaries for air and one inlet boundary for fuel. The velocity profiles for both air flows are extracted from grid-based CFD predictions and imposed as boundary conditions. In Fig. 4a and 4b the shape of the velocity profiles imposed at each inlet is presented. The axial velocity profile of the fuel flow at the inlet is based on a correlation for laminar flows. At the fuel inlet, the circumferential velocity recovers a free vortex with sharp velocity gradients at the sides. This profile is used in order to mimic an established annular flow with no slip condition at 


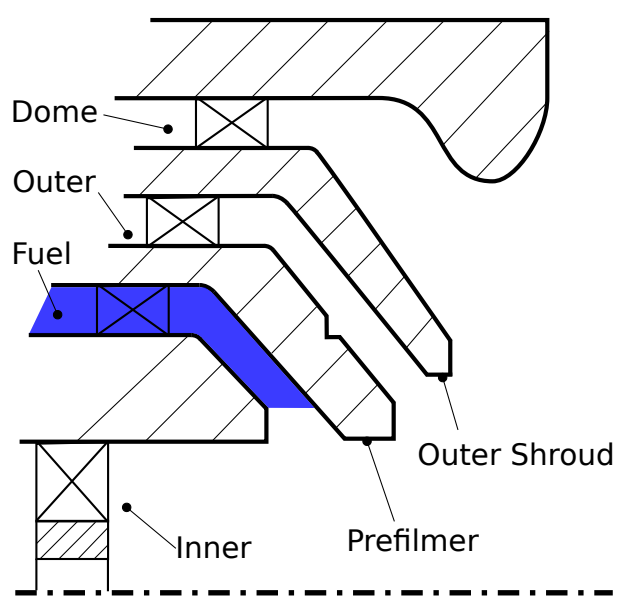

(a) Geometry

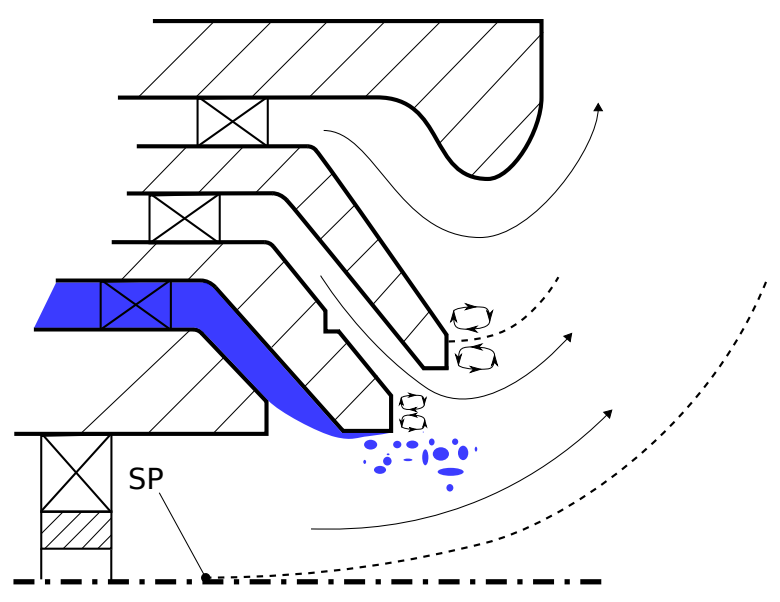

(b) Local Air Flow

Figure 2. Fuel Spray Nozzle

Table 1. Thermodynamic Conditions and Fluid Properties

\begin{tabular}{cccc} 
& & Air & Fuel \\
\hline $\mathrm{T}$ & $\mathrm{K}$ & 649 & 293.15 \\
$\rho$ & $\mathrm{kg} \mathrm{m}^{-3}$ & 6.51 & 770 \\
$\mu$ & $10^{-3} \mathrm{Pas}$ & 0.03255 & 1.56 \\
\hline
\end{tabular}

the walls. The pressure gradient is set to zero at all inlet boundaries. At the outlet cross-section of the domain, the pressure is set constant and the velocity gradient in normal direction is imposed to be zero.

In order to capture the influence of vortex breakdown, the boundaries of the stream tube containing the inner and outer air are extracted from grid based one-phase flow predictions and imposed as slip walls in the SPH setup as depicted in Fig. $3 a$.

The thermodynamic conditions of the underlying operating point are presented in Tab. 1. Surface tension coefficients are set for the liquid to recover static contact angles of $60^{\circ}$. The pressure is $1.213 \mathrm{MPa}$ and the surface tension $27.510^{-3} \mathrm{~N} \mathrm{~m}^{-1}$.

As exemplified in Fig. 3b, the cross-section of the fuel duct at the inlet constitutes an annulus. Further downstream, the duct is deflected in radial direction and forced to smaller radii. Whenever the main direction of the flow exhibits a radial component, the cross-section constitutes a frustoconical surface. The area of the frustoconical surface scales with the radius. The same effect applies to the outer air duct.

The presented setup enables the computation of the local multi-phase flow in the vicinity of the atomizer at a high spatial resolution of $10 \mu \mathrm{m}$. The 3D sector covers 5 degrees. We are aware of the fact, that in order to resolve details of the flow in circumferential direction, the size of the segment is too small. However, swirl effects can inherently be captured. Furthermore, the sector recovers the inherently three dimensional shape of the flow cross-section along the flow path as illustrated in Fig. 3b.

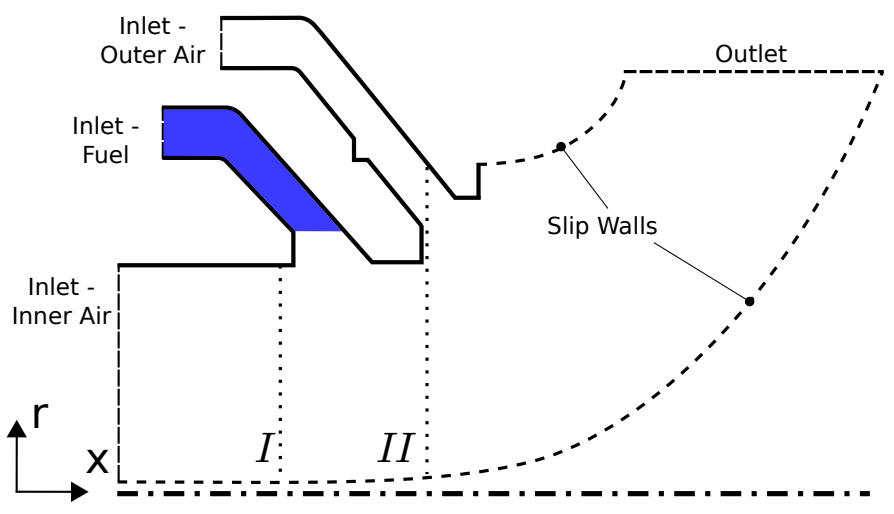

(a) Boundary Conditions

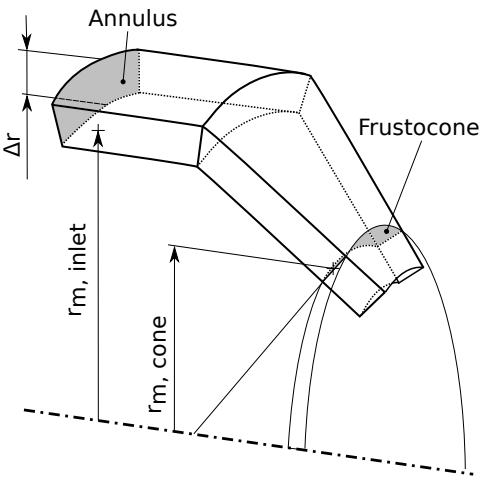

(b) Cross-Sections in Fuel Duct

Figure 3. Computational Domain

Aiming at lower computational costs, predictions based on two-dimensional domains are desired. However, 2D 


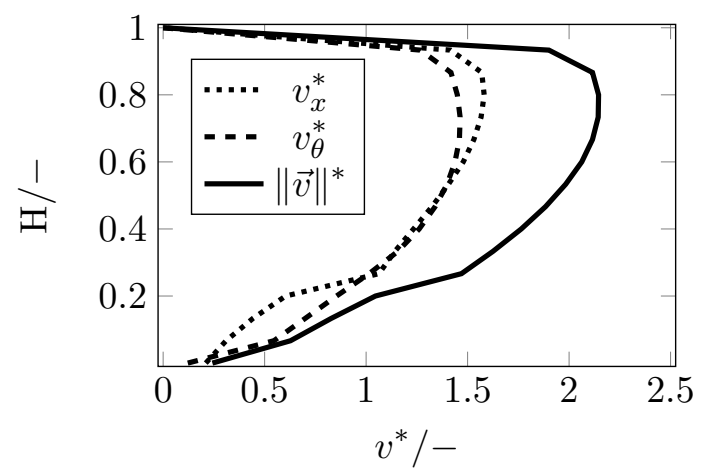

(a) Inner Air Duct

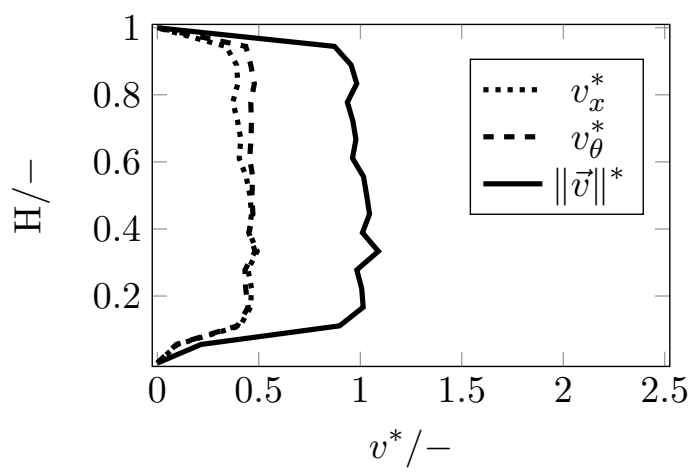

(b) Outer Air Duct

Figure 4. Inlet Boundary Conditions

predictions violate the inherently three dimensional character of fuel atomization. They furthermore imply a planar extension in lateral direction, also violating the frustoconical shape of the flow cross-sections as presented before. In order to identify and quantify differences between 2D and 3D predictions, 2D computations are also performed. In 2D the circumferential velocity at the inlet of each duct is used as input to the 2D swirl model. All the other boundary conditions are kept constant. A discretization study in 2D up to a resolution of $2.5 \mu \mathrm{m}$ has been conducted, demonstrating that a spatial resolution of at least $10 \mu \mathrm{m}$ is required.

\section{Results and Discussion}

\section{Gaseous Flow - Velocity Profiles}

In Fig. 5a the non-dimensional velocity profiles of the axial velocity in $2 \mathrm{D}$ and $3 \mathrm{D}$ are depicted at the axial position I. The co-ordinate $\mathrm{H}$ is the non-dimensional channel height. The non-dimensional channel height is 1 at the radius of the prefilmer lip. As to be expected, the shape of the profiles is similar to the shape of the axial velocity imposed at the inlet boundary. Obviously, the maximum of the axial velocity component in 2D is located at a larger radius than in the inherently three dimensional case. As the flow is not developed at position I, it comes to a radial velocity component. Comparing 2D and 3D, larger values of the radial velocity can be stated in case of the 2D prediction. Both, the shift of the maximum of the axial velocity and the larger value of the radial velocity indicate that the virtual centrifugal forces seem to be slightly stronger than the inherent centrifugal forces in 3D. The 2D swirl model is based on the assumption of conserved angular momentum. Contrary, in 3D angular momentum is not conserved because of friction losses. Hence, lower centrifugal forces in 3D are to be expected.

In Fig. $5 \mathrm{c}$ the resulting velocity magnitudes in $2 \mathrm{D}$ and $3 \mathrm{D}$ are depicted. In $2 \mathrm{D}$ only a representative centrifugal force is modeled, but the circumferential velocity component is not present. Therefore, there is no contribution to the magnitude of the absolute velocity $\|v\|^{*}$. Hence, the magnitude of the velocity is smaller in $2 \mathrm{D}$ compared to 3D. The velocity distribution of air at position I acts on the liquid fuel flow exiting the fuel gallery. The centrifugal forces of the inner air flow lead to a force in radial direction and, hence, to an interaction with the fuel flow inside the exit of the fuel duct.

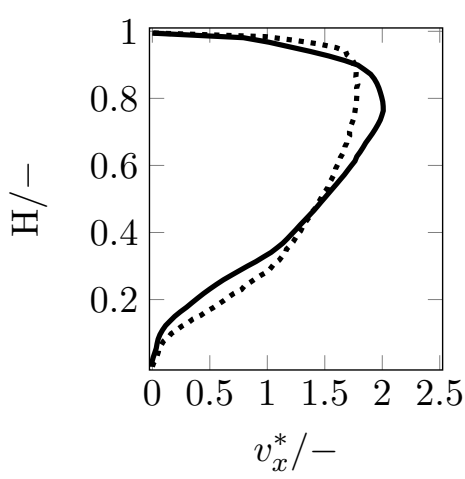

(a) Axial Velocity

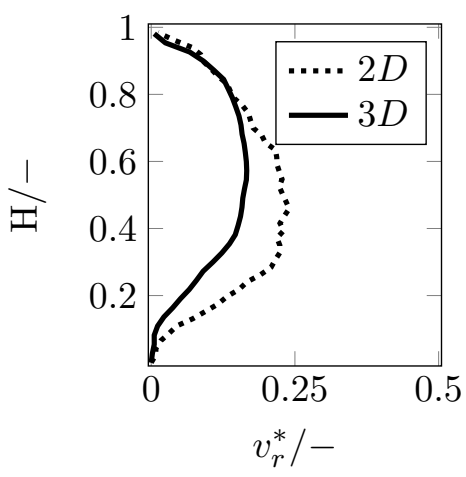

(b) Radial Velocity

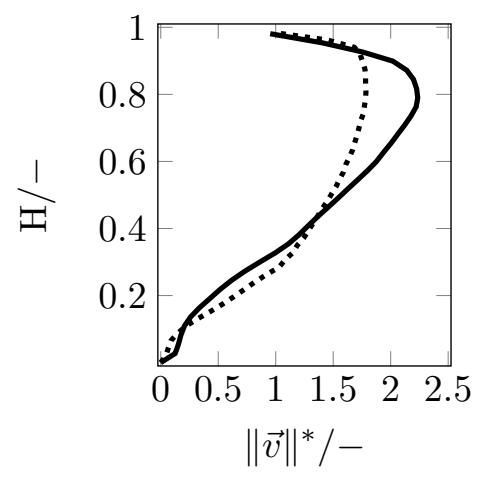

(c) Velocity Magnitude

Figure 5. Comparison of Velocity Profiles at Position I - 2D vs. 3D

In Fig. 6 the velocity profiles at position II obtained from 2D and 3D predictions are presented. In contrast to the inner air flow, the differences of the velocity magnitude in the outer air flow are significantly larger. Again in 2D there is no contribution of the circumferential velocity to the magnitude. Furthermore, the flow is accelerated according to the continuity equation because of the decrease of the flow cross-section as illustrated in Fig. 3b. In 2D this effect is 
omitted. Hence, only a deflection of the flow, but no acceleration takes place resulting in a much smaller magnitude of the velocity.

One can conclude from the velocity profiles, that deviations of the gaseous flow between 2D and 3D occur. The differences inside the inner air duct are comparably small. The largest differences are to be expected downstream the prefilming surface in the vicinity of the shear zone between inner and outer air.

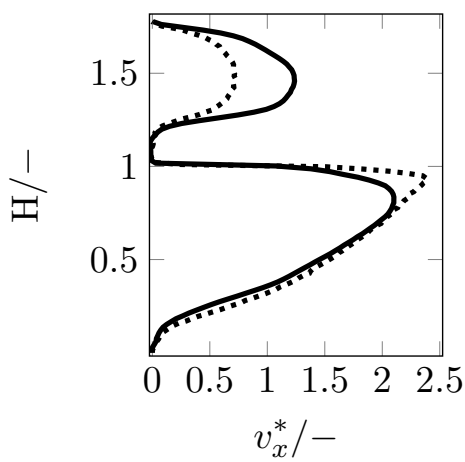

(a) Axial Velocity

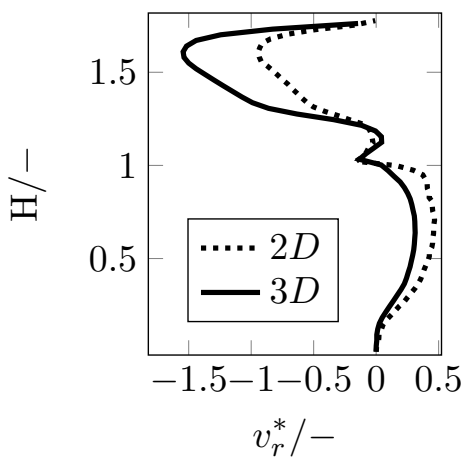

(b) Radial Velocity

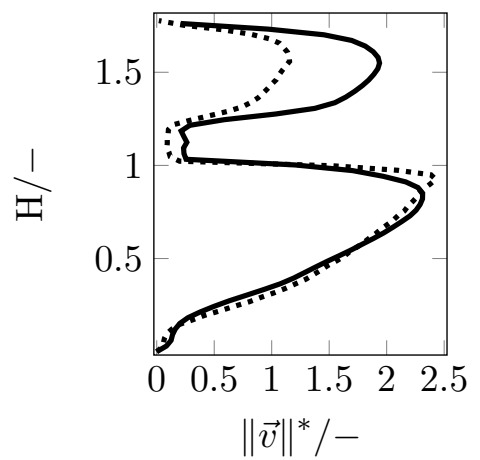

(c) Velocity Magnitude

Figure 6. Comparison of Velocity Profiles - 2D vs. 3D - Position II

\section{Gaseous Flow - Flow Instabilities}

Due to the Lagrangian nature of the SPH method, a color coding can easily be applied, which serves as a tool to distinguish between inner and outer air flow. Fuel is colored in blue and air originating from the outer air inlet is indicated as orange. Air that entered the domain through the inner air inlet is not visible. By means of the color coding the Kelvin-Helmholtz instability between inner and outer air flow can be visualized. Two instantaneous snapshots are presented in Fig. 7. The snapshots are recorded at the beginning of the simulation, when there is no fuel emerging from the supply duct. Despite the differences with regard to the velocity field in the outer air duct, the Kelvin-Helmholtz instability is qualitatively captured in 2D and 3D. Because of the higher velocity in the outer air duct in 3D, the shear zone is located at a smaller radius than in 2D. In each case, two vortices occur in the snapshots. The fuel exiting the fuel gallery will be released into the corresponding shear zone and exposed to the local flow instabilities of the gaseous flow. The Kelvin-Helmholtz instability presented here is one example of a transient flow instability that might occur in the vicinity of the nozzle. Instabilities in circumferential direction might also occur, but just a subset of wavelengths is captured in the present numerical setup due to the small circumferential extension. Later in time, as soon as fuel emerges from the supply duct, the distinct vortices due to the Kelvin-Helmholtz instability will vanish. However, instabilities of the gaseous flow in radial direction still occur and interact with the liquid.
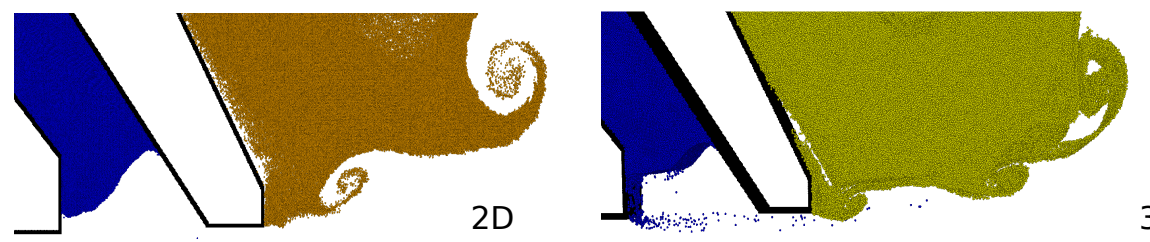

Figure 7. Comparison of Kelvin-Helmholtz Instability

\section{Liquid Flow}

The design of the air flow with fuel emerging from the fuel gallery, leads to a periodic filling and discharge effect at the exit region of the fuel duct. In Fig. 8 three snapshots presenting the different phases of the periodic discharge are depicted. In the beginning fuel is accumulated in the exit region of the fuel duct. Once the cavity is filled up with fuel, a ligament is formed reaching out into the inner air duct. The momentum of the inner air flow pushes the ligament against the prefilming surface. The ligament is released into the shear zone and breaks up into smaller ligaments. Once the ligament is extracted, the fill level of the cavity reaches its minimum. Then for a period of time fuel is again accumulated in the cavity, before the formation of a new ligament starts again. In 3D and 2D the same periodic effect can be observed.

In Fig. 9 the time evolution of liquid volume flow rate across the circular cross-section of the inner air duct at axial position II is depicted. The periodic discharge effect is recovered in terms of periodic peaks of the liquid volume flow rate. Facing comparably large computational effort in 3D, only a few discharge events are captured. Comparing $2 \mathrm{D}$ and $3 \mathrm{D}$, one can conclude from the time histories that the peaks do not coincide in time. The reason for the 


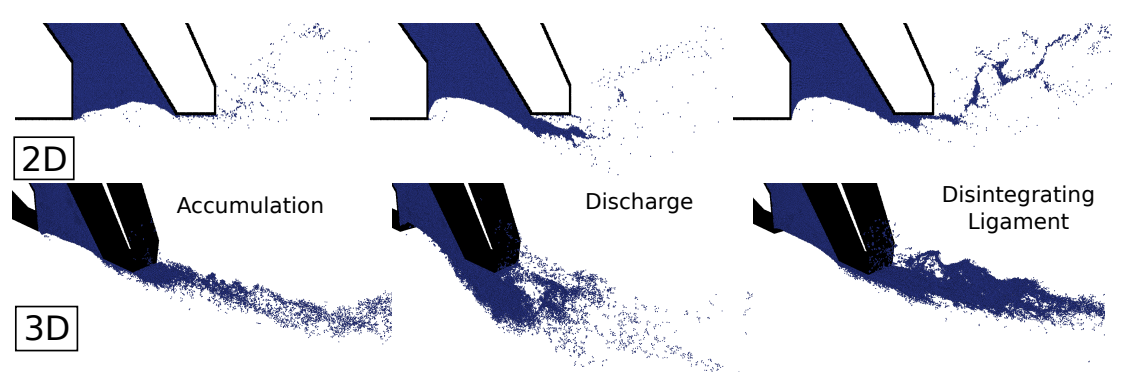

Figure 8. Periodic Filling and Discharge

difference is the higher velocity of fuel at the outlet of the fuel gallery in 3D due to the reduced frustoconical crosssection compared to 2D. Based on the few available events, the frequency of the periodic discharge is determined to be approximately $1.6 \mathrm{kHz}$. However, the number of events is far below statistical significance.

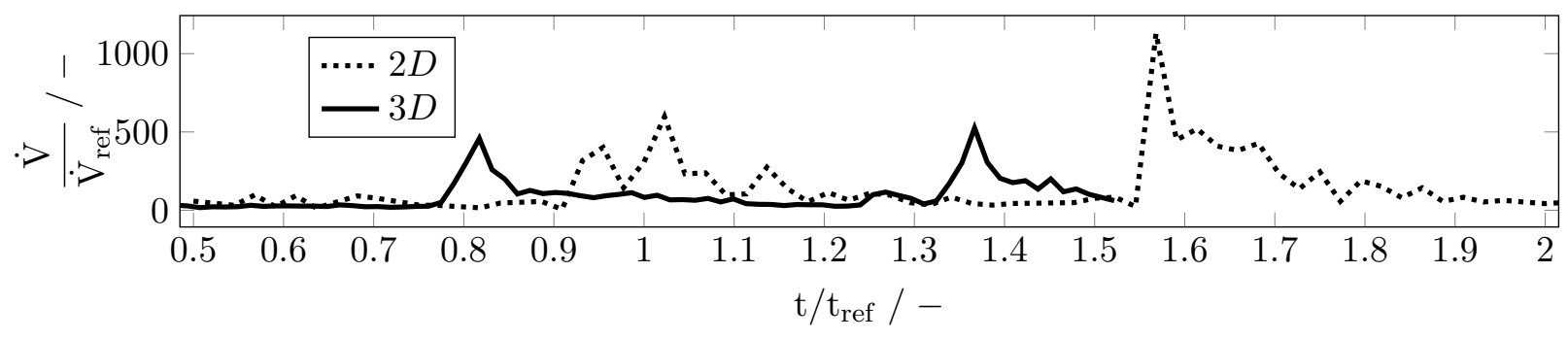

Figure 9. Evolution of the Non-Dimensional Volume Flow Rate over Time

\section{Conclusions}

A methodology for the numerical analysis of primary breakup by means of SPH in the vicinity of fuel spray nozzles at typical engine conditions is presented. A typical airblast-nozzle for aero-engines with three air flow ducts is analyzed. Predictions based on a 3D sector are performed and compared to predictions based on simplified two dimensional setups. To the knowledge of the authors this is the first analysis of primary breakup in a fuel spray nozzle at engine conditions in 3D by means of SPH. The following conclusions can be drawn:

1. SPH is suitable for the analysis of primary breakup phenomena in realistic fuel spray nozzle configurations.

2. Unsteady effects in the gaseous and the liquid flow field are identified.

3. Temporal fluctuations and flow instabilities can be studied in 3D, which resemble those observed in experimental data.

4. Large quantitative discrepancies between 2D and 3D simulations occur.

5. However, 2D simulations are capable of giving qualitative insights into local, unsteady flow phenomena.

\section{Acknowledgements}

This work was performed on the computational resource ForHLR Phase II funded by the Ministry of Science, Research and Arts Baden-Württemberg and DFG ("Deutsche Forschungsgemeinschaft"). In addition the authors would like to thank Rolls-Royce Deutschland Ltd \& Co KG for the outstanding cooperation. The authors also are grateful for many lively and fruitful discussions with Simon Holz.

\section{Nomenclature}

\section{Abbreviations}

ITS Institut für Thermische Strömungsmaschinen

PDF Probability Density Function

SMD Sauter Mean Diameter

SPH Smoothed Particle Hydrodynamics

SP Stagnation Point

VoF Volume of Fluid

\section{Latin Symbols}

$c \quad$ Speed of Sound $\left[\mathrm{m} \mathrm{s}^{-1}\right]$

$d \quad$ Diameter [m]

$h \quad$ Smoothing Length $[\mathrm{m}]$

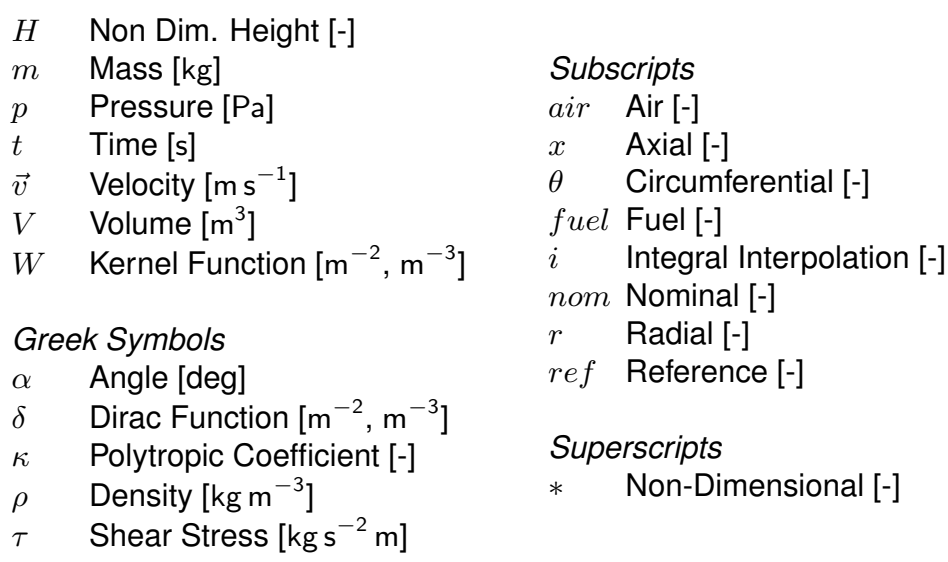




\section{References}

[1] S. Braun, M. Krug, L. Wieth, C. Höfler, R. Koch, and H.-J. Bauer, "Simulation of primary atomization: Assessment of the smoothed particle hydrodynamics (sph) method," in 13th Triennial International Conference on Liquid Atomization and Spray Systems (ICLASS), Tainan, Taiwan, 2015.

[2] S. Braun, L. Wieth, R. Koch, and H.-J. Bauer, "Influence of trailing edge height on primary atomization: Numerical studies applying the smoothed particle hydrodynamics (sph) method," in 13th Triennial International Conference on Liquid Atomization and Spray Systems, Tainan. Taiwan., 2015.

[3] T. Dauch, S. Braun, W. L., G. Chaussonnet, M. Keller, R. Koch, and B. H.-J., "Computation of liquid fuel atomization and mixing by means of the SPH method: application to a jet engine fuel nozzle," ASME 2016 Turbo Expo: Turbine Technical Conference and Exposition, Seoul, 2016.

[4] R. Koch, S. Braun, L. Wieth, G. Chaussonnet, T. Dauch, and H.-J. Bauer, "Prediction of primary atomization using smoothed particle hydrodynamics," European Journal of Mechanics - B/Fluids, vol. 61, Part 2, pp. 271 278, 2017. Rotating Flows.

[5] C. Höfler, S. Braun, R. Koch, and H.-J. Bauer, "Modeling spray formation in gas turbines-a new meshless approach," Journal of Engineering for Gas Turbines and Power, vol. 135, pp. 011503-011503, Nov. 2012.

[6] M. C. Keller, S. Braun, L. Wieth, G. Chaussonnet, T. Dauch, R. Koch, C. Höfler, and H.-J. Bauer, "Numerical modeling of oil-jet lubrication for spur gears using smoothed particle hydrodynamics," in Proceedings of the 11th International SPHERIC Workshop, pp. 69-76, 2016.

[7] G. Chaussonnet, O. Vermorel, E. Riber, and B. Cuenot, "A new phenomenological model to predict drop size distribution in large-eddy simulations of airblast atomizers," International Journal of Multiphase Flow, vol. 80, pp. 29-42, Apr 2016.

[8] R. Gingold and J. Monaghan, "Smoothed particle hydrodynamics-theory and application to non-spherical stars," Monthly notices of the royal astronomical society, vol. 181, pp. 375-289, 1977.

[9] L. Lucy, "A numerical approach to the testing of the fission hypothesis," The astronomical journal, vol. 82, pp. 1013-1024, 1977.

[10] G. Chaussonnet, S. Braun, L. Wieth, R. Koch, and H.-J. Bauer, "Influence of particle disorder and smoothing length on sph operator accuracy," in 10th International SPHERIC Workshop, Tainan, Taiwan, 2015.

[11] J. Swegle, D. Hicks, and S. Attaway, "Smoothed particle hydrodynamics stability analysis," Journal of Computational Physics, vol. 116, no. 1, pp. 123 - 134, 1995.

[12] M. Liu and G. Liu, "Smoothed particle hydrodynamics (sph): an overview and recent developments," Archives of Computational Methods in Engineering, vol. 17, no. 1, pp. 25-76, 2010.

[13] N. Quinlan, M. Basa, and M. Lastiwka, "Truncation error in mesh-free particle methods," International Journal for Numerical Methods in Engineering, vol. 66, p. 2064-2085, 2006.

[14] S. Litvinov, X. Hu, and N. Adams, "Towards consistence and convergence of conservative sph approximations," Journal of Computational Physics, vol. 301, pp. 394-401, Nov. 2015.

[15] W. Dehnen and H. Aly, "Improving convergence in smoothed particle hydrodynamics simulations without pairing instability," Monthly Notices of the Royal Astronomical Society, vol. 425, no. 2, p. 1068, 2012.

[16] L. Wieth, C. Lieber, W. Kurz, S. Braun, R. Koch, and B. H.-J., "Numerical modeling of an aero-engine bearing chamber using the meshless smoothed particle hydrodynamics method," in Proceedings of ASME Turbo Expo 2015: Turbine Technical Conference and Exposition, Montréal, Canada, GT2015-42316, vol. 2B, 2015.

[17] A. Colagrossi and M. Landrini, "Numerical simulation of interfacial flows by smoothed particle hydrodynamics," Journal of Computational Physics, vol. 191, no. 2, pp. 448 - 475, 2003.

[18] J. J. Monaghan, "Smoothed particle hydrodynamics," Annual Review of Astronomy and Astrophysics, vol. 30, no. 1, pp. 543-574, 1992.

[19] K. Szewc, J. Pozorski, and J.-P. Minier, "Analysis of the incompressibility constraint in the smoothed particle hydrodynamics method," International Journal for Numerical Methods in Engineering, vol. 92, no. 4, pp. 343369, 2012.

[20] G. Batchelor, An introduction to fluid dynamics. Cambridge University Press, 2000.

[21] L. Wieth, S. Braun, R. Koch, and H.-J. Bauer, "Modeling of liquid-wall interaction using the meshless smoothed particle hydrodynamics (sph) method," in ILASS Europe, 26th Annual Conference on Liquid Atomization and Spray Systems, Bremen, Germany, 2014.

[22] S. Adami, X. Hu, and N. Adams, "A new surface-tension formulation for multi-phase $\{\mathrm{SPH}\}$ using a reproducing divergence approximation," Journal of Computational Physics, vol. 229, no. 13, pp. 5011 - 5021, 2010.

[23] D. Violeau and B. D. Rogers, "Smoothed particle hydrodynamics (sph) for free-surface flows: past, present and future," Journal of Hydraulic Research, vol. 54, no. 1, pp. 1-26, 2016.

[24] A. Mansour and M. Benjamin, "Pure airblast nozzle." U.S. Patent US6622488 B2, September 2003. 